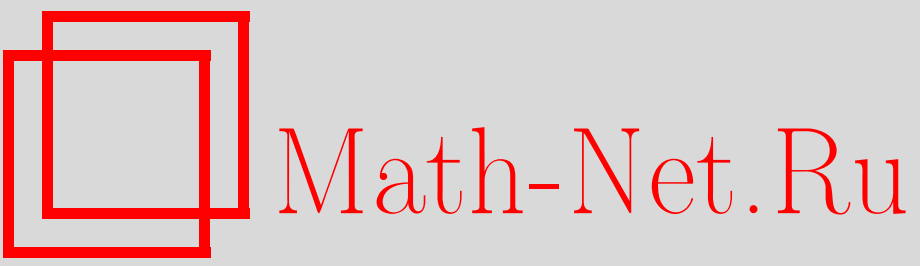

О. И. Мохов, О согласованных потенциальных деформациях фробениусовых алгебр и уравнениях ассоциативности, УМН, 1998, том 53, выпуск 2, 153-154

DOI: https://doi.org/10.4213/rm40

Использование Общероссийского математического портала Math-Net.Ru подразумевает, что вы прочитали и согласны с пользовательским соглашением

http://www.mathnet.ru/rus/agreement

Параметры загрузки:

IP: 54.84 .234 .179

26 апреля 2023 г., 08:48:47 


\title{
О СОГЛАСОВАННЫХ ПОТЕНЦИАЛЬНЫХ ДЕФОРМАЦИЯХ ФРОБЕНИУСОВЫХ АЛГЕБР И УРАВНЕНИЯХ АССОЦИАТИВНОСТИ
}

\author{
О.И. Мохов
}

Пусть $\eta^{i j}$ - произвольная невырожденная постоянная симметричная матрица: $\eta^{i j}=\eta^{j i}$, $\eta^{i j}=$ const, $\operatorname{det}\left(\eta^{i j}\right) \neq 0,1 \leqslant i, j \leqslant N$. Рассмотрим функцию $N$ переменных $F\left(t^{1}, \ldots, t^{N}\right)$, удовлетворяющую следующему условию: функции $c_{j k}^{i}(t)=\eta^{i s} \partial^{3} F / \partial t^{s} \partial t^{j} \partial t^{k}$ для всех $t=$ $\left(t^{1}, \ldots, t^{N}\right)$ задают структурные константы ассоциативной алгебры $A(t)$ в $N$-мерном линейном пространстве с базисом $e_{1}, \ldots, e_{N}$ и умно жением $e_{i} \circ e_{j}=c_{i j}^{k}(t) e_{k}$. Условие ассоциативности $\left(e_{i} \circ e_{j}\right) \circ e_{k}=e_{i} \circ\left(e_{j} \circ e_{k}\right)$, которое эквивалентно соотношению $c_{i j}^{s} c_{s k}^{l}=$ $c_{i s}^{l} c_{j k}^{s}$ для структурных констант алгебры $A(t)$, приводит к сложной переопределенной системе нелинейных уравнений в частных производных третьего порядка для функции $F$ :

$$
\eta^{s r} \frac{\partial^{3} F}{\partial t^{s} \partial t^{i} \partial t^{j}} \frac{\partial^{3} F}{\partial t^{k} \partial t^{l} \partial t^{r}}=\eta^{s r} \frac{\partial^{3} F}{\partial t^{s} \partial t^{i} \partial t^{k}} \frac{\partial^{3} F}{\partial t^{j} \partial t^{l} \partial t^{r}} .
$$

Система (1) впервые возникла в двумерной топологической теории поля и известна как система Виттена-Дийкграафа-Г. Верлинде-Е. Верлинде-Дубровина или уравнения ассоциативности (см. [1]-[3]). При любом $t$ алгебра $A(t)$ является коммутативной ассоциативной алгеброй с заданной на ней невырожденной симметричной билинейной формой $\left\langle e_{i}, e_{j}\right\rangle=\eta_{i j}$, которая является инвариантной относительно умножения в алгебре, т.е. $\left\langle e_{i} \circ e_{j}, e_{k}\right\rangle=\left\langle e_{i}, e_{j} \circ e_{k}\right\rangle\left(\eta_{i s} \eta^{s j}=\delta_{i}^{j}\right.$ по определению). Кроме того, обычно требуют, чтобы элемент $e_{1}$ всегда являлся единицей в алгебре $A(t)$, что эквивалентно дополнительному условию $\eta_{i j}=\partial^{3} F / \partial t^{1} \partial t^{i} \partial t^{j}$ (см. [1]-[3]), но мы в данной статье откажемся от этого требования (см. также [4]-[6]). Конечномерная коммутативная ассоциативная алгебра с заданной на ней невырожденной инвариантной симметричной билинейной формой назьвается алгеброй Фробениуса (отметим, что обычно дополнительно требуют наличия единицы в алгебре Фробениуса). Соответственно, функции $F(t)$, являющиеся решениями уравнений ассоциативности (1), описывают $N$-параметрические деформации $N$-мерных алгебр Фробениуса, которые назьваются потенциальными, а функция $F(t)$ называется потенииалом деформации. Рассмотрим теперь функцию тех же $N$ переменных $\Phi\left(t^{1}, \ldots, t^{N}\right)$, удовлетворяющую следующим двум условиям:

1) гессиан этой функции невырожден, т.е. $\mu_{i j}\left(t^{1}, \ldots, t^{N}\right)=\partial^{2} \Phi / \partial t^{i} \partial t^{j}, \operatorname{det}\left(\mu_{i j}\right) \neq 0$, $\mu^{i s} \mu_{s j}=\delta_{j}^{i}$

2) для любого $t=\left(t^{1}, \ldots, t^{N}\right)$ функиии $b_{j k}^{i}\left(t^{1}, \ldots, t^{N}\right)=\mu^{i s}(t) \partial^{3} \Phi / \partial t^{s} \partial t^{j} \partial t^{k}$ задают структурные константы ассочиативной алгебры $L(t)$ в $N$-мерном линейном пространстве с базисом $e_{1}, \ldots, e_{N}$ и умножением $e_{i} * e_{j}=b_{i j}^{k}(t) e_{k}$.

Условие ассоциативности $\left(e_{i} * e_{j}\right) * e_{k}=e_{i} *\left(e_{j} * e_{k}\right)$ приводит к системе нелинейных уравнений

$$
\mu^{s r}(t) \frac{\partial^{3} \Phi}{\partial t^{s} \partial t^{i} \partial t^{j}} \frac{\partial^{3} \Phi}{\partial t^{k} \partial t^{l} \partial t^{r}}=\mu^{s r}(t) \frac{\partial^{3} \Phi}{\partial t^{s} \partial t^{i} \partial t^{k}} \frac{\partial^{3} \Phi}{\partial t^{j} \partial t^{l} \partial t^{r}} .
$$

При любом $t$ алгебра $L(t)$ является коммутативной ассоциативной алгеброй с заданной на ней невырожденной симметричной билинейной формой $\left(e_{i}, e_{j}\right)=\mu_{i j}(t)$, которая инвариантна относительно умножения в алгебре: $\left(e_{i} * e_{j}, e_{k}\right)=\left(e_{i}, e_{j} * e_{k}\right)$. Таким образом, функции $\Phi(t)$, являющиеся решениями системы (2), также описьвают $N$-параметрические деформации $N$-мерных алгебр Фробениуса.

ПримеР. В случае $N=2$ система (2) эквивалентна уравнению

(3) $\Phi_{22}\left(\Phi_{111} \Phi_{122}-\Phi_{112}^{2}\right)+\Phi_{12}\left(\Phi_{112} \Phi_{122}-\Phi_{111} \Phi_{222}\right)+\Phi_{11}\left(\Phi_{112} \Phi_{222}-\Phi_{122}^{2}\right)=0$, где введены естественные обозначения, используемые всюду далее в статье: $\Phi_{i j}=\partial^{2} \Phi / \partial t^{i} \partial t^{j}$, $\Phi_{i j k}=\partial^{3} \Phi / \partial t^{i} \partial t^{j} \partial t^{k}$. Уравнение (3), а также система (2) при любом $N,-$ интегрируемы.

Работа выполнена при частичной финансовой поддержке РФФИ (грант № 96-01-01623), РФФИ-ННИО (грант № 96-01-00050G) и INTAS (грант № 96-0770). 
ПреДЛОЖЕНИЕ. В общем случае произвольного $N$ уравнения ассочиативности (2) әквивалентны условиям совместности для следующей линейной системы:

$$
\partial \Psi_{i} / \partial t^{j}=z b_{i j}^{k}(t) \Psi_{k}(t), \quad 1 \leqslant i, j, k \leqslant N,
$$

где $b_{i j}^{k}(t)=\mu^{k s}(t) \Phi_{s i j}(t), z=$ const - произвольный параметр.

Для системы (1) аналогичное утверждение (для структурных констант $c_{j k}^{i}(t)$ ) доказано Дубровиным в [3].

На алгебре $L(t)$ кроме билинейной формы $\left(e_{i}, e_{j}\right)=\mu_{i j}(t)$ определена еще одна невырожденная симметричная билинейная форма, а именно $\left\langle e_{i}, e_{j}\right\rangle=\eta_{i j}$. Аналогично, на алгебре $A(t)$ кроме билинейной формы $\left\langle e_{i}, e_{j}\right\rangle=\eta_{i j}$ определена невырожденная симметричная билинейная форма $\left(e_{i}, e_{j}\right)=\mu_{i j}(t)$.

ТеоРема 1. Если форма $\eta_{i j}$ является инвариантной на алгебре $L(t)$, m.e.

$$
\left\langle e_{i} * e_{j}, e_{k}\right\rangle=\left\langle e_{i}, e_{j} * e_{k}\right\rangle,
$$

то функция $\Phi(t)$ удовлетворяет системе (1). Аналогично, если форма $\mu_{i j}(t)$ является инвариантной на алгебре $A(t)$ (это условие эквивалентно условию (5)), то функция $F(t)$ удовлетворяет системе (2). В обоих этих случаях уравнения ассочиативности (1) и (2) әквивалентны.

Условие (5) эквивалентно системе нелинейных уравнений на функцию $\Phi(t)$ :

$$
\eta^{s r} \Phi_{r i} \Phi_{s j k}=\eta^{s r} \Phi_{r k} \Phi_{s j i} .
$$

Это условие играет важную роль в задаче классификации согласованных пуассоновых структур гидродинамического типа (см. [7]), и мы будем называть его условием согласованной потенчиальной деформачии фробениусовых алгебр $A(t)$ и $L(t)$.

ТЕОРема 2. Функция $\Phi(t)$, являющаяся решением нелинейных систем (2) и (6), порождает пару согласованных гамильтоновых операторов гидродинамического типа $\left(\right.$ здесь $\left.t^{i}=t^{i}(x), i=1, \ldots, N\right)$ :

$$
K^{i j}=\eta^{i j} d / d x, \quad M^{i j}=\eta^{i s} \eta^{j r} \Phi_{s r}(t(x)) d / d x+1 / 2 \eta^{i s} \eta^{j r} \Phi_{s r k}(t(x)) t_{x}^{k} .
$$

Теорема 3. Пусть $N=2 u \eta^{i j}=\varepsilon^{i} \delta^{i j}$, где $\varepsilon^{i}= \pm 1, i=1,2$. Тогда функиия $\Phi(t)$ является решением нелинейных систем (2) и (6) тогда и только тогда, когда әта функция $\Phi(t)$ является решением линейного уравнения в частных производных второго порядка

$$
\alpha\left(\varepsilon^{1} \Phi_{11}-\varepsilon^{2} \Phi_{22}\right)=\beta \Phi_{12},
$$

где $\alpha$ и $\beta$ - произвольнье константы.

\section{СПИСОК ЛИТЕРАТУРЫ}

[1] Witten E. // Surv. Diff. Geom. 1991. V. 1. P. 243-310. [2] Dijkgraaf R., Verlinde H., Verlinde E. // Nucl. Phys. 1991. V. B 352. P. 59-86. [3] Dubrovin B. // Lect. Notes Math. 1996. V. 1620. P. 120-348; hep-th/9407018. [4] Mokhov O. I. // Geometry from the Pacific Rim. Proc. Pacific Rim Geometry Conf. National Univ. Singapore, December 12-17, 1994. Berlin: Walter de Gruyter, 1997. P. 285-309. [5] Ferapontov E. V., Mokhov O. I. // Proc. First Int. Workshop "Nonlinear Physics". Gallipoli (Lecce), Italy. 29 June - 7 July, 1995. Singapore: World Scientific, 1996. Р. 104-115. [6] Мохов О. И. Дис. ... докт. физ.-матем. наук. М.: МИРАН, 1996. [7] Мохов О. И. // УМН. 1997. Т. 52. № 6. С. 173-174.

Центр нелинейных исследований при Институте теоретической физики им. Л. Д. Ландау РАН 